\title{
SUMMABILITY OF ALTERNATING GAP SERIES
}

\author{
J. P. KEATING ${ }^{1}$ AND J. B. READE ${ }^{2}$ \\ ${ }^{1}$ School of Mathematics, University of Bristol, Bristol BS8 $1 T W$, UK and \\ BRIMS, Hewlett-Packard Laboratories, Filton Road, \\ Stoke Gifford, Bristol BS12 6QZ, UK \\ ${ }^{2}$ Department of Mathematics, The University of Manchester, Manchester M13 9PL, UK
}

(Received 15 December 1997; revised 14 April 1999)

\begin{abstract}
The Abel and Cesàro summabilities of two alternating gap series are investigated. We prove that the series $\sum_{n=0}^{\infty}(-1)^{n} x^{n^{2}}$ is summable at $x=1$ (in both senses), but that $\sum_{n=0}^{\infty}(-1)^{n} x^{2^{n}}$ is not. In 1907, Hardy obtained essentially the same result for the latter series; our proof is shorter and more elementary: we use the Poisson summation formula to derive an explicit estimate for the size of the oscillations as $x \rightarrow 1_{-}$. This represents an example of a general method for determining the Abel summability of similar series.
\end{abstract}

Keywords: summability; Poisson summation; gap series

AMS 1991 Mathematics subject classification: Primary 4 OC99

\section{Introduction}

Our purpose here is to illustrate some subtleties concerning the summability at $x=1$ of alternating gap series of the form

$$
\sum_{n=0}^{\infty}(-1)^{n} x^{\alpha(n)}
$$

where $\alpha(n)$ is an increasing sequence of non-negative integers. We call such a series Abel summable to $A$ if

$$
\lim _{x \rightarrow 1} \sum_{n=0}^{\infty}(-1)^{n} x^{\alpha(n)}
$$

exists and takes the finite value $A$. For example, when $\alpha(n)=n$,

$$
\sum_{n=0}^{\infty}(-1)^{n} x^{n}=\frac{1}{1+x} \rightarrow \frac{1}{2}
$$

as $x \rightarrow 1_{-}$, and so the series is Abel summable to $A=\frac{1}{2}$. 
Likewise, writing

$$
\sum_{n=0}^{\infty}(-1)^{n} x^{\alpha(n)}=\sum_{k=0}^{\infty} a_{k} x^{k}
$$

where

$$
a_{k}= \begin{cases}(-1)^{n}, & \text { if } k=\alpha(n), \\ 0, & \text { otherwise, }\end{cases}
$$

and defining the $m$ th partial sum of the coefficients by

$$
s_{m}=\sum_{k=0}^{m} a_{k}
$$

we call the series (1.1) Cesàro summable to $C$ when $x=1$ if

$$
\lim _{n \rightarrow \infty} \frac{1}{n} \sum_{m=0}^{n-1} s_{m}
$$

exists and takes the finite value $C$. In the case $\alpha(n)=n, s_{2 m}=1$ and $s_{2 m+1}=0$, and so $C=\frac{1}{2}(=A)$.

The summability of gap series has been investigated before, for example by Hardy [3]. Our main aim in this paper is to re-examine the question of Abel summability using an approach based on the Poisson summation formula. To motivate this, in $\S 2$ we discuss two gap series that exhibit different summability properties. The first, with $\alpha(n)=n^{2}$, is shown to be summable (in both senses). The second has $\alpha(n)=2^{n}$ and is not summable. In $\S 3$, we determine its asymptotic form as $x \rightarrow 1_{-}$explicitly, using the Poisson summation formula. This illustrates a general method for dealing with similar series.

\section{Summability}

We begin by noting the implications for gap series of two well-known facts about summability. First, if the series (1.1) is Cesàro summable to $S$, this implies that it is also Abel summable to $S$ (see, for example, [3, Theorem 55]). (Note that this covers the example discussed in $\S 1$, for which $\alpha(n)=n$.) Second, if the series is Abel summable to $S$, and if the partial sums are bounded (i.e. $s_{m}=O(1)$ ), this implies that the series is also Cesàro summable to $S[\mathbf{3}$, Theorem 92].

We now consider the following two examples in the light of these general results.

Example 2.1 $\left(\alpha(n)=n^{2}\right)$. In this case,

$$
y_{n}=\frac{s_{0}+s_{1}+\cdots+s_{n-1}}{n}
$$


takes alternate maxima and minima at $n=k^{2}$. The maxima are

$$
\begin{aligned}
y_{k^{2}} & =\frac{k^{2}-(k-1)^{2}+(k-2)^{2}-(k-3)^{2}+\cdots+1}{k^{2}} \\
& =\frac{1}{2} \frac{k+1}{k}
\end{aligned}
$$

for $k$ odd. The minima are

$$
\begin{aligned}
y_{k^{2}} & =\frac{(k-1)^{2}-(k-2)^{2}+(k-3)^{2}-(k-4)^{2}+\cdots+1}{k^{2}} \\
& =\frac{1}{2} \frac{k-1}{k},
\end{aligned}
$$

for $k$ even. Therefore, taking the limit as $k \rightarrow \infty$, the series is Cesàro summable with $C=\frac{1}{2}$. It then follows from the first of the results quoted above that it is also Abel summable with $A=\frac{1}{2}$.

Example $2.2\left(\alpha(n)=2^{n}\right)$. In this case, $y_{n}$ takes alternate maxima and minima at $n=2^{k}$. The maxima are

$$
\begin{aligned}
y_{2^{k}} & =\frac{2^{k}-2^{k-1}+2^{k-2}-2^{k-3}+\cdots+2-1}{2^{k}} \\
& =\frac{1}{3} \frac{2^{k+1}-1}{2^{k}}
\end{aligned}
$$

for $k$ odd; and the minima are

$$
\begin{aligned}
y_{2^{k}} & =\frac{2^{k-1}-2^{k-2}+2^{k-3}-2^{k-4}+\cdots+2-1}{2^{k}} \\
& =\frac{1}{3} \frac{2^{k}-1}{2^{k}},
\end{aligned}
$$

for $k$ even. Therefore, the limit as $k \rightarrow \infty$ does not exist: $y_{n}$ has maxima whose heights tend to $\frac{2}{3}$, and minima whose heights tend to $\frac{1}{3}$. Hence the series is not Cesàro summable. It then follows from the second of the results quoted above that neither can it be Abel summable (because the partial sums $s_{m}$ are bounded). Hardy gave a direct proof of this in [2]. The question we address here is: what is the asymptotic form of the gap series (1.1) in this case as $x \rightarrow 1 \_$?

\section{Poisson summation}

The Poisson summation formula for a function $f \in L^{1}(\mathbb{R})$ with Fourier transform

$$
\hat{f}(u)=\int_{-\infty}^{\infty} \mathrm{e}^{\mathrm{i} t u} f(t) \mathrm{d} t
$$


is

$$
\sum_{n=-\infty}^{\infty} f(n)=\sum_{n=-\infty}^{\infty} \hat{f}(2 \pi n)
$$

which is valid if, for example, the sum on the right-hand side is absolutely convergent and

$$
\sum_{k=-\infty}^{\infty} f(x+k)
$$

converges absolutely and uniformly in $x$ (see [4, p. 129]).

Our main purpose in this paper is to demonstrate how the Poisson summation formula can be used to investigate the Abel summability of gap series. To do this, we now apply it to the two examples discussed in the previous section.

We note first that it follows from (3.2) that

$$
\sum_{n=-\infty}^{\infty}(-1)^{n} f(n)=\sum_{n=-\infty}^{\infty} \hat{f}((2 n+1) \pi)
$$

and it is this form that we shall make direct use of.

Example 3.1 $\left(\alpha(n)=n^{2}\right)$. Consider the function $f(t)=x^{t^{2}}=\mathrm{e}^{-\lambda t^{2}}$, where $x=$ $\mathrm{e}^{-\lambda}(\lambda>0)$. The Fourier transform of $f(t)$ is

$$
\hat{f}(u)=\int_{-\infty}^{\infty} \mathrm{e}^{\mathrm{i} t u} \mathrm{e}^{-\lambda t^{2}} \mathrm{~d} t=\sqrt{\frac{\pi}{\lambda}} \mathrm{e}^{-u^{2} / 4 \lambda} .
$$

Applying the Poisson summation formula in the form (3.4) thus gives

$$
\sum_{n=-\infty}^{\infty}(-1)^{n} x^{n^{2}}=\sqrt{\frac{\pi}{\lambda}} \sum_{n=-\infty}^{\infty} \mathrm{e}^{-(2 n+1)^{2} \pi^{2} / 4 \lambda}
$$

validity being assured by the convergence of the series on the right-hand side, and the uniform convergence over $t \in \mathbb{R}$ of the series

$$
\phi(t)=\sum_{n=-\infty}^{\infty}(-1)^{n} \mathrm{e}^{-\lambda(t+n)^{2}}
$$

It follows that

$$
\begin{aligned}
\sum_{n=0}^{\infty}(-1)^{n} x^{n^{2}} & =\frac{1}{2}+\sqrt{\frac{\pi}{\lambda}} \sum_{n=0}^{\infty} \mathrm{e}^{-(2 n+1)^{2} \pi^{2} / 4 \lambda} \\
& \rightarrow \frac{1}{2}
\end{aligned}
$$


as $x \rightarrow 1_{-}, \lambda \rightarrow 0_{+}$since

$$
\begin{aligned}
\sqrt{\frac{\pi}{\lambda}} \sum_{n=0}^{\infty} \mathrm{e}^{-(2 n+1)^{2} \pi^{2} / 4 \lambda} & <\sqrt{\frac{\pi}{\lambda}} \sum_{n=1}^{\infty} \mathrm{e}^{-n^{2} \pi^{2} / 4 \lambda} \\
& <\sqrt{\frac{\pi}{\lambda}} \sum_{n=1}^{\infty} \mathrm{e}^{-n \pi^{2} / 4 \lambda} \\
& =\sqrt{\frac{\pi}{\lambda}} \frac{\mathrm{e}^{-\pi^{2} / 4 \lambda}}{1-\mathrm{e}^{-\pi^{2} / 4 \lambda}} \\
& \rightarrow 0
\end{aligned}
$$

as $\lambda \rightarrow 0_{+}$. Hence, as already proved in $\S 2$, in this case the gap series is Abel summable to $A=\frac{1}{2}$, coinciding with the value of the Cesàro summation.

Example 3.2 $\left(\alpha(n)=2^{n}\right)$. Consider the function $f(t)=x^{2^{|t|}}=\mathrm{e}^{-\lambda 2^{|t|}}$. Its Fourier transform is

$$
\begin{aligned}
\hat{f}(u) & =\int_{-\infty}^{\infty} \mathrm{e}^{\mathrm{i} t u} \mathrm{e}^{-\lambda 2^{|t|}} \mathrm{d} t \\
& =2 \operatorname{Re} \int_{0}^{\infty} \mathrm{e}^{\mathrm{i} t u} \mathrm{e}^{-\lambda 2^{t}} \mathrm{~d} t \\
& =\frac{2}{\log 2} \operatorname{Re} \frac{1}{\lambda^{\mathrm{i} u / \log 2}} \int_{\lambda}^{\infty} s^{(\mathrm{i} u / \log 2)-1} \mathrm{e}^{-s} \mathrm{~d} s
\end{aligned}
$$

putting $s=\lambda 2^{t}$. Now, clearly, for all real $u \neq 0$,

$$
\begin{aligned}
\int_{\lambda}^{\infty} s^{(\mathrm{i} u / \log 2)-1} \mathrm{e}^{-s} \mathrm{~d} s & =\Gamma(\mathrm{i} u / \log 2)-\int_{0}^{\lambda} s^{(\mathrm{i} u / \log 2)-1} \mathrm{e}^{-s} \mathrm{~d} s \\
& =\Gamma(\mathrm{i} u / \log 2)-\sum_{k=0}^{\infty} \frac{(-1)^{k}}{k !} \frac{\lambda^{(\mathrm{i} u / \log 2)+k}}{(\mathrm{i} u / \log 2)+k}
\end{aligned}
$$

where the last equality follows by expanding the exponential in powers of $s$ and integrating term by term.

Poisson's summation formula (3.4) thus gives

$$
\begin{aligned}
& \sum_{n=-\infty}^{\infty}(-1)^{n} x^{2^{|n|}} \\
& =\frac{2}{\log 2} \operatorname{Re} \sum_{n=-\infty}^{\infty}\left(\frac{\Gamma((2 n+1) \pi \mathrm{i} / \log 2)}{\lambda^{(2 n+1) \pi \mathrm{i} / \log 2}}-\sum_{k=0}^{\infty} \frac{(-1)^{k}}{k !} \frac{\lambda^{k}}{((2 n+1) \pi \mathrm{i} / \log 2)+k}\right)
\end{aligned}
$$


and, hence,

$$
\begin{aligned}
\sum_{n=0}^{\infty}(-1)^{n} x^{2^{n}}=\frac{1}{2} x+\frac{2}{\log 2} \operatorname{Re} \sum_{n=0}^{\infty}\left(\frac{\Gamma((2 n+1) \pi \mathrm{i} / \log 2)}{\lambda^{(2 n+1) \pi \mathrm{i} / \log 2}}\right. & \left.-\sum_{k=0}^{\infty} \frac{(-1)^{k}}{k !} \frac{\lambda^{k}}{((2 n+1) \pi \mathrm{i} / \log 2)+k}\right) .
\end{aligned}
$$

Observe, firstly, that

$$
\begin{aligned}
\operatorname{Re} \sum_{n=0}^{\infty} \sum_{k=0}^{\infty} \frac{(-1)^{k}}{k !} \frac{\lambda^{k}}{((2 n+1) \pi \mathrm{i} / \log 2)+k} & =\sum_{n=0}^{\infty} \sum_{k=0}^{\infty} \frac{(-1)^{k}}{k !} \frac{k \lambda^{k}}{((2 n+1) \pi / \log 2)^{2}+k^{2}} \\
& =\sum_{k=1}^{\infty} \frac{(-1)^{k} \lambda^{k}}{(k-1) !} \sum_{n=0}^{\infty} \frac{1}{((2 n+1) \pi / \log 2)^{2}+k^{2}} \\
& \rightarrow 0,
\end{aligned}
$$

as $\lambda \rightarrow 0_{+}$(that is, as $x \rightarrow 1_{-}$).

Observe, secondly, that if we write $\lambda=2^{-\mu}$, then

$$
\frac{2}{\log 2} \operatorname{Re} \sum_{n=0}^{\infty} \frac{\Gamma((2 n+1) \pi \mathrm{i} / \log 2)}{\lambda^{(2 n+1) \pi \mathrm{i} / \log 2}}=\frac{2}{\log 2} \operatorname{Re} \sum_{n=0}^{\infty} \Gamma((2 n+1) \pi \mathrm{i} / \log 2) \mathrm{e}^{(2 n+1) \mu \pi \mathrm{i}}
$$

is 2-periodic in $\mu=-(1 / \log 2) \log \log (1 / x)$. Furthermore, using the fact (see, for example, $[\mathbf{1}$, p. 256]) that

$$
|\Gamma(\mathrm{i} y)|^{2}=\frac{\pi}{y \sinh (\pi y)}
$$

for real $y$, the first term of (3.15) oscillates with amplitude

$$
\begin{aligned}
\frac{2}{\log 2}|\Gamma(\pi \mathrm{i} / \log 2)| & =\frac{2}{\sqrt{\log 2 \sinh \left(\pi^{2} / \log 2\right)}} \\
& =2.75 \times 10^{-3},
\end{aligned}
$$

to three significant figures, and the sum of the rest is bounded in modulus by

$$
\begin{aligned}
\frac{2}{\log 2} \sum_{n=1}^{\infty}\left|\Gamma\left(\frac{(2 n+1) \pi \mathrm{i}}{\log 2}\right)\right| & =\frac{2}{\sqrt{\log 2}} \sum_{n=1}^{\infty} \frac{1}{\sqrt{(2 n+1) \sinh \left((2 n+1) \pi^{2} / \log 2\right)}} \\
& <\frac{2}{\sqrt{\log 2}} \frac{1}{\sqrt{3 \sinh \left(3 \pi^{2} / \log 2\right)}} \sum_{m=0}^{\infty} \mathrm{e}^{-m \pi^{2} / \log 2} \\
& =\frac{2}{\sqrt{\log 2}} \frac{1}{\sqrt{3 \sinh \left(3 \pi^{2} / \log 2\right)}} \frac{1}{1-\mathrm{e}^{-\pi^{2} / \log 2}} \\
& =1.04 \times 10^{-9},
\end{aligned}
$$

to three significant figures. 
It follows that as $x \rightarrow 1$ - the series $\sum_{n=0}^{\infty}(-1)^{n} x^{2^{n}}$ oscillates round $x=\frac{1}{2}$ with amplitude $2.75 \times 10^{-3}$, to three significant figures, and is asymptotically 2-periodic in $\mu=$ $-(1 / \log 2) \log \log (1 / x)$. Hence, one can choose values of $x$ arbitrarily close to 1 for which

$$
\sum_{n=0}^{\infty}(-1)^{n} x^{2^{n}}=\frac{1}{2} \pm\left(2.75 \times 10^{-3}\right),
$$

to three significant figures. This is, therefore, a direct proof of the fact that the series is not Abel summable, as was already deduced in $\S 2$ from the fact that it is not Cesàro summable. Clearly, the method based on Poisson summation allows one to go further, in that it provides an answer to the question raised at the end of that section: it gives the asymptotic form of the gap series as $x \rightarrow 1_{-}$. Interestingly, in this particular example, the values of the maxima and minima of the gap series as $x \rightarrow 1_{-}$do not coincide with the maxima and minima of the partial Cesàro averages in (1.7) as $n \rightarrow \infty$.

\section{References}

1. M. Abramowitz AND I. A. Stegun, Handbook of mathematical functions (Dover, New York, 1970).

2. G. H. HARDY, Q. J. Math. 38 (1907), 269-288.

3. G. H. HARDY, Divergent series (Oxford University Press, 1949).

4. Y. KATZNELSON, An introduction to harmonic analysis (Wiley, Jerusalem, 1968). 